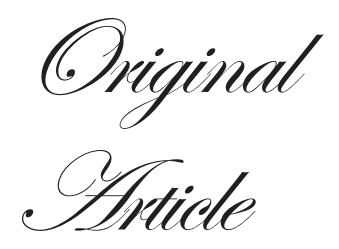

\title{
Role of endoscopic band ligation in management of non-variceal upper gastrointestinal bleeding
}

\author{
Arunkumar Krishnan, Vimalraj Velayutham, Jeswanth Satyanesan, \\ Selvakumar Eswaran, Surendran Rajagopal
}

\section{ABSTRACT}

Centre for GI bleed \& Institute of Surgical Gastroenterology and Liver Transplant, Stanley Medical College Hospital, Chennai, India

\section{Correspondence:}

Dr. Arunkumar Krishnan

Email: dr.arunkumarpillai@ gmail.com
Background: Acute non-variceal upper GI bleeding (NVUGIB) is a challenging emergency condition. Early endoscopic therapy has been recommended as the first-line of treatment for upper GI bleeding (UGIB) as it has been shown to reduce recurrent bleeding. We aimed to determine the various causes of NVUGIB and discuss the role of band ligation.

Patients and methods: A total of 74 patients with NVUGIB who had been treated with endoscopic band ligation (EBL), between November 2006 and December 2011, were included in the study. Bleeding lesions included Dieulafoy lesion (DL), Mallory-Weiss tears (MWTs), duodenal ulcer, post-surgical anastomosis bleed and gastric ulcer after polypectomy. After the basic life support was provided, all patients underwent emergent and elective endoscopy. Results: The study comprised 49 (66.2\%) men and 25 (33.8\%) women. The mean age was $48.2 \pm 6.4$ years for men and $40.6 \pm 2.2$ years for women. MWTs and DL constituted the majority of bleeding lesions, of these 26 and 17, respectively required EBL. Other causes were: prepyloric ulcer 11; duodenal ulcer 9; ulcers in antrum 5; post-polypectomy bleed 3; anastomosis bleed 1; and malignant lesions 2. Bleeding stopped after endoscopic therapy in $96.5 \%$ of patients. The single failure was in bleeding from a pre-pyloric lesion which was treated by using injection sclerotherapy with 1:10,000 adrenaline solution where EBL was not successful. Conclusion: EBL provides safe and effective modality for haemostasis in patients with NVUGIB. EBL could be considered as an alternative method of choice for treatment of endoscopic haemostasis in patients with NVGIB.

KEYWORDS: Non-variceal upper gastrointestinal bleeding (NVUGIB), endoscopic haemostasis, endoscopic band ligation (EBL)

\section{Introduction}

Acute non-variceal upper GI bleeding (NVUGIB) is an emergency case and a frequent clinical problem. NVUGIB occurs in 40-150 cases per 100,000 populations. Despite improvements in endoscopic and supportive therapies, the overall mortality rate due to NVUGIB remains at around $10 \%$, and may even reach $35 \%$ in hospitalized patients with serious comorbidity. ${ }^{1}$ Early endoscopic therapy has generally been recommended as the first-line of treatment for upper GI bleeding (UGIB) as it has been shown to reduce recurrent bleeding, the need for surgery and mortality for patients with high-risk NVUGIB; thus endoscopic haemostasis has become the treatment of choice. ${ }^{2}$ In addition to the various endoscopic techniques used for GI bleeding, such as injection with or without thermal therapy, mechanical methods such as the use

(C) Tropical Gastroenterology 2013 
of haemoclip and band ligation have recently been used. Endoscopic band ligation (EBL) is now a well-established therapeutic modality with documented efficacy and safety in the treatment of oesophageal variceal bleeding. ${ }^{3}$ It is unclear whether there is any benefit of ligation over other endoscopic techniques for NVUGIB. This prospective study aimed to determine the various causes of NVUGIB and discusses the role of band ligation.

\section{Material and methods}

A total of 74 patients with NVUGIB who had been treated with EBL, between November 2006 and December 2011, were included in the present study. Patients who had some form of endoscopic haemostasis for NVUGIB were excluded from the study. Bleeding lesions included Dieulafoy lesion (DL), MalloryWeiss tears (MWTs), duodenal ulcer, post-surgical anastomosis bleed and gastric ulcer after polypectomy. After providing the basic life support, all patients underwent emergent and elective endoscopy. For patients admitted with GI bleeding, intravenous fluid resuscitation and proton pump inhibitor (PPI) therapy was performed, and if necessary, blood was transfused. Vital signs were recorded. Routine rectal digital examination and nasogastric irrigation were performed. In case of clear nasogastric drainage, the tube was removed. Active bleeding and presence of haematemesis were indications for emergent endoscopy. Patients with no clinical evidence of active bleeding had endoscopy performed within 24 hours of admission.

Fujinon EG-450WR5 endoscope (Fujinon Optical Co Ltd, Tokyo, Japan) systems were used for endoscopy. After identifying the bleeding site and the lesion, EBL was performed with the single seed Multiple Band Ligator (Wilson-Cook medical, Winston-Salem NC, USA). The bleeding lesion was drawn into the banding device with suction, and then the rubber band was placed around the bleeding point. In fibrotic and hard mucosal tissue, although suction is successful, the band may later release from the lesion. Therefore, in the present study the only inclusion criteria for EBL were the characteristics of the lesion. Lesions taken up for EBL were superficial and non-fibrotic. Unsuccessful haemostasis was defined as recurrent bleeding from the treatment site within 12 hours of the primary haemostatic treatment. ${ }^{4}$ Patients with active bleeding at presentation and those in whom the basic treatment was thought suboptimal had to repeat endoscopic therapy within 24-48 hours. Patients without recurrent bleeding after endoscopic treatment underwent endoscopy on the 2nd and 14th days after the basic haemostatic treatment for follow-up. An intervention was considered successful on achieving haemostasis under direct endoscopic visualization. After the endoscopic therapy, intravenous PPI therapy was continued in all the patients. Oral feeding was started after 24 hours for patients who had no evidence of recurrent bleeding. The study was approved by the institute ethics committee.

\section{Results}

An overview of the data collected for the study is shown in Table 1. A total of 74 patients underwent EBL therapy for NVUGIB, among them 49 (66.2\%) were men and 25 (33.8\%) were women. The mean age was $48.2 \pm 6.4$ years for men and 40.6 \pm 2.2 years for women. Cases of MWTs and DL constituted the majority of bleeding lesions (26 [35.1\%] and 17 [23\%], respectively) requiring EBL. Other causes were: pre-pyloric ulcer 11 (14.9\%); duodenal ulcer 9 (12.2\%); ulcers in antrum 5 (6.7\%); post-polypectomy bleeds 3 (4\%); anastomosis bleed 1 $(1.3 \%)$; and malignant lesions $2(2.7 \%)$.

During endoscopy, active bleeding such as oozing or a pulsatile artery was seen for $43(58.1 \%)$ of the lesions compared to $20(27 \%)$ showing a non-bleeding visible vessel and 11 (14.9\%) showing an adherent clot. Bleeding stopped after the endoscopic therapy in $96.5 \%$ of patients. No patient in this group had a previous history of UGIB. Twenty-one (24.4\%) patients were haemodynamically unstable at the time of admission and 14 (18.9\%) patients required blood transfusion. Blood was seen in nasogastric drainage in 8 patients $(10.8 \%)$; digital examination of the rectum revealed the presence of melaena in these patients.

Except one, all patients had complete haemostasis, and rebleeding did not occur. The only patient in whom the rebleed occurred had a pre-pyloric ulcer which required endoscopic injection of 1:10,000 solution of epinephrine intervention due

\section{Table 1: Overview of data}

\begin{tabular}{lc}
\hline Aetiology of bleeding & Number of patients (\%) \\
\hline Mallory-Weiss tear & $26(35.1)$ \\
Dieulafoy lesion & $17(23)$ \\
Pre-pyloric ulcer & $11(14.9)$ \\
Duodenal ulcer & $9(12.2)$ \\
Ulcers in antrum & $5(6.7)$ \\
Malignant lesions & $2(2.7)$ \\
Post-polypectomy bleed & $3(4)$ \\
Anastomosis bleed & $1(1.3)$ \\
\hline
\end{tabular}


to coagulopathy caused by active liver disease. EBL was performed without any complications. Follow-up endoscopic examinations after 2 days and 3 weeks revealed healed scar tissues replacing the original bleeding pathologies. The mean follow-up was 29 months (range 7-46 months). During the outpatient follow-up, there was no recurrence of bleeding and there were no procedure-related complications. Emergency surgery was not required for any patient. There was no mortality in this series.

\section{Discussion}

Endoscopy has become a safe and effective first-line therapy for control of UGIB. Developed in the 1980s,EBL has emerged as the method of choice for the treatment of oesophageal variceal bleeding. EBL is indicated for bleeding lesions without fibrosis because the lesion and surrounding tissue must be aspirated into the hood of the tip of the endoscope for successful ligation. ${ }^{5}$

Endoscopic therapy is indicated for patients with actively bleeding or spurting arterial vessels and for those with a nonbleeding visible vessel in an ulcer. Clean-based ulcers have an extremely low rate of recurrent bleeding and do not require endoscopic treatment. The presence of blood in the upper GI tract, active spurting haemorrhage and a 'non-bleeding visible vessel' are signs of poor prognosis.

MWTs are laceration at or near the oesophagogastric junction that may cause acute GI bleed. They account for 5\%$15 \%$ of cases of UGIB. Fortunately, a majority of MWTs resolve spontaneously, but $1 \%-3 \%$ require intervention. ${ }^{6}$ In a prospective, randomized trial comparing EBL with endoscopic injection therapy (EIT) for bleeding MWTs, a total of 34 patients were enrolled; 17 patients underwent EBL and 17 patients underwent EIT. Primary haemostasis was achieved in all the 17 patients in the EBL group and 16 in the EIT group. There was no difference in the efficacy or safety between the two groups. EBL was successful in all 10 patients. Among them, 4 patients required blood transfusions before endoscopic treatment. ${ }^{6}$

DLs are extremely rare causes of GI bleeding. The erosion of the mucosa and the arterial wall can initiate massive bleeding. DL is also an infrequent cause of UGIB, accounting for $0.3 \%-$ $6.7 \%$ of cases. ${ }^{7}$ Effective EBL of a bleeding rectal DL that was previously treated with haemoclip has also been reported. ${ }^{8}$ Mumtaz et al. ${ }^{9}$ analysed the outcome of patients with DL who were treated with EBL compared to those treated with thermal injection therapy. Active haemostasis was achieved in all the patients in both the groups but early rebleeding occurred in those in the thermal injection therapy group. The authors concluded that EBL is as effective in the treatment of DL as injection with or without thermal therapy. The risk of rebleeding for a conservatively treated DL is reported to be $15 \%$. However, none of the patients had a rebleed, and none required surgery after initial EBL in DL. Surgical therapy is reported to be required in only $4 \%-8 \%$ of patients with DL. ${ }^{10-12}$ It has been shown that EBL and haemoclip are equally effective and safe for the treatment of active bleeding in MWTs ${ }^{12}$ and DL. ${ }^{4}$ Postpolypectomy haemorrhage occurs in $0.5 \%-2.2 \%$ of cases of endoscopic polypectomy and may be delayed. ${ }^{13}$ Few case reports mentioned initial haemostasis with EBL following polypectomy. ${ }^{14,15}$

Peptic ulcer bleeding accounts for $28 \%-59 \%$ of all episodes of NVUGIB. ${ }^{16}$ The American Society of Gastrointestinal Endoscopy (ASGE) guidelines state that no single modality was shown to be superior for treating UGIB caused by peptic ulcer disease. ${ }^{16}$ Park et al. ${ }^{4}$ reported 19 patients with major stigmata of acute peptic ulcer bleed who were treated using EBL-11 patients had active bleeding and 8 patients had a non-bleeding visible vessel. The site of peptic ulcer was gastric in 12 patients, duodenum in 4 patients and Billroth II anastomoses in 3 patients. Initial haemostasis was achieved in a single session in all the patients. The authors concluded that EBL was an effective and safe endoscopic treatment for smallsized, non-fibrotic peptic ulcers. In our series, one patient had a rebleed and required EIT to control the bleed after initial EBL due to a large ulcer $(2.5 \mathrm{~cm})$.

Only one patient with a pre-pyloric ulcer was managed with additional EIT to control active bleeding because of coagulopathy. All patients were discharged without any complications. EBL devices, commonly used in variceal bleeding, have been used to treat non-variceal causes of bleeding. The procedure is relatively simple and the equipment required is readily available and inexpensive compared to other costly mechanical devices such as haemoclip.

In conclusion, EBL provides a safe and effective modality for haemostasis in NVUGIB. It could be considered an alternative method of choice for the treatment of endoscopic haemostasis in patients with NVUGIB due to various pathologies. Large, controlled, prospective studies comparing quality and cost-effectiveness of band ligation with other endoscopic methods are required before this modality is put to wide use. 


\section{References}

1. Barkun A, Sabbah S, Enns R, Armstrong D, Gregor J, Fedorak $\mathrm{RN}$, et al. The Canadian Registry on Nonvariceal Upper Gastrointestinal Bleeding and Endoscopy (RUGBE): endoscopic hemostasis and proton pump inhibition are associated with improved outcomes in a real-life setting. Am J Gastroenterol. 2004;99:1238-46.

2. Cook DJ, Gayatt GH, Salena BJ, Laine LA. Endoscopic therapy for acute nonvariceal upper gastrointestinal hemorrhage: a metaanalysis. Gastroenterology. 1992;102:139-48.

3. Laine L, Cook D. Endoscopic ligation compared with sclerotherapy for treatment of esophageal variceal bleeding. A meta-analysis. Ann Intern Med. 1995;123:280-7.

4. Park CH, Lee WS, Joo YE, Choi SK, Rew JS, Kim SJ. Endoscopic band ligation for control of acute peptic ulcer bleeding. Endoscopy. 2004;36:79-82.

5. Abi-Hanna D, Williams SJ, Gillespie PE, Bourke MJ. Endoscopic band ligation for non-variceal non-ulcer gastrointestinal hemorrhage. Gastrointest Endosc. 1998;48:510-14.

6. Park CH, Min SW, Sohn YH, Lee WS, Joo YE, Kim HS, et al. A prospective, randomized trial of endoscopic band ligation vs. epinephrine injection for actively bleeding Mallory-Weiss syndrome. Gastrointest Endosc. 2004;60:22-7.

7. Reilly HF 3rd, al-Kawas FH. Dieulafoy's lesion. Diagnosis and management. Dig Dis Sci. 1991;36:1702-7.

8. Yoshikumi Y, Mashima H, Suzuki J, Yamaji Y, Okamoto M, Ogura $\mathrm{K}$, et al. A case of rectal Dieulafoy's ulcer and successful endoscopic band ligation. Can J Gastroenterol. 2006;20:287-90.

9. Mumtaz R, Shaukat M, Ramirez FC. Outcomes of endoscopic treatment of gastroduodenal Dieulafoy's lesion with rubber band ligation and thermal/injection therapy. J Clin Gastroenterol. 2003;36:310-14.

10. Yarze JC. Routine endoscopic 'marking' of Dieulafoy-like lesions. Am J Gastroenterol. 2001;96:264-5.

11. Norton ID, Petersen BT, Sorbi D, Balm RK, Alexander GL, Gostout CJ. Management and long-term prognosis of Dieulafoy lesion. Gastrointest Endosc. 1999;50:762-7.

12. Cho YS, Chae HS, Kim HK, Kim JS, Kim BW, Kim SS, et al. Endoscopic band ligation and endoscopic hemoclip placement for patients with Mallory-Weiss syndrome and active bleeding. World J Gastroenterol. 2008;14:2080-4.

13. Rex DK, Lewis BG, Waye JD. Colonoscopy and endoscopic therapy for delayed post-polypectomy haemorrhage. Gastrointest Endosc. 1992;38:127-9.

14. Slivka A, Parsons WG, Carr-Locke DL. Endoscopic band ligation for treatment of post-polypectomy hemorrhage. Gastrointest Endosc. 1994;40:230-2.

15. Smith RE, Doull J. Treatment of colonic post-polypectomy bleeding site by endoscopic band ligation. Gastrointest Endosc. 1994;40:499-500.

16. Adler DG, Lei ghton JA, Davila RE, Hirota WK, Jacobson BC, Qureshi WA, et al. American Society of Gastrointestinal Endoscopy (ASGE). Guideline: the role of endoscopy in acute non-variceal upper-GI hemorrhage. Gastrointes Endosc. 2004;60:497-504. 